\title{
Validity domain and limitation of non-retarded Green's tensor for electromagnetic scattering at surfaces
}

\author{
Philippe Gay-Balmaz *, Olivier J.F. Martin \\ Electromagnetic Fields and Microwave Electronics Laboratory, Swiss Federal Institute of Technology, ETH-Zentrum, ETZ, \\ CH-8092 Zurich, Switzerland
}

Received 13 June 2000; accepted 2 August 2000

\begin{abstract}
This work gives a detailed derivation of the non-retarded dyadic Green's tensor associated with surfaces in the quasistatic approximation. The derivation is made from a rigorous model where the dyadic is expressed as Sommerfeld integrals. We then assess the domain where this approximation can be used for scattering calculations on surfaces by comparing rigorous and non-retarded solutions. Implications of this work for scattering calculations in near-field optics are finally discussed. (C) 2000 Elsevier Science B.V. All rights reserved.
\end{abstract}

\section{Introduction}

Many scattering problems are successfully analyzed using Green's tensor technique, also known as coupled-dipole approximation (CDA) or discrete-dipole approximation (DDA). These problems range from scattering by interstellar dust to the optical properties of metallic spheres in suspension [1-5].

The core of this technique is the Fredholm equation of second kind for the electric field $\mathbf{E}(\mathbf{r})$ scattered by the three-dimensional (3D) scatterer of relative permittivity $\varepsilon(\mathbf{r})$ illuminated with the incident field $\mathbf{E}^{0}(\mathbf{r})$ :

\footnotetext{
${ }^{*}$ Corresponding author. Fax: +41-1-632-1198.

E-mail addresses: balmaz@ifh.ee.ethz.ch (P. Gay-Balmaz), martin@ifh.ee.ethz.ch (O.J.F. Martin).
}

$\mathbf{E}(\mathbf{r})=\mathbf{E}^{0}(\mathbf{r})+\int_{V} \mathrm{~d} \mathbf{r}^{\prime} \mathbf{G}\left(\mathbf{r}, \mathbf{r}^{\prime}\right) \cdot k_{0}^{2} \Delta \varepsilon\left(\mathbf{r}^{\prime}\right) \mathbf{E}\left(\mathbf{r}^{\prime}\right)$,

where $\mathbf{G}\left(\mathbf{r}, \mathbf{r}^{\prime}\right)$ is the Green's tensor associated with the background where the scatterer is placed and $k_{0}$, the vacuum wave number. Throughout the paper, we assume time harmonic fields with an $\exp (-\mathrm{i} \omega t)$ dependence.

Eq. (1) is not limited to scattering by objects in infinite homogeneous space, but can also handle scatterers placed on a surface or embedded in a stratified background, as depicted in Fig. 1. In this case, Green's tensor $\mathbf{G}\left(\mathbf{r}, \mathbf{r}^{\prime}\right)$ must account for this complex background and the dielectric contrast between the scatterer and the surrounding medium depends on the location of the scatterer: $\Delta \varepsilon(\mathbf{r})=\varepsilon(\mathbf{r})-\varepsilon_{2}$ when it is in the upper layer and $\Delta \varepsilon(\mathbf{r})=\varepsilon(\mathbf{r})-\varepsilon_{1}$ when it is confined to the lower layer (throughout the paper, we assume nonmagnetic backgrounds: $\mu=\mu_{0}$ ). 


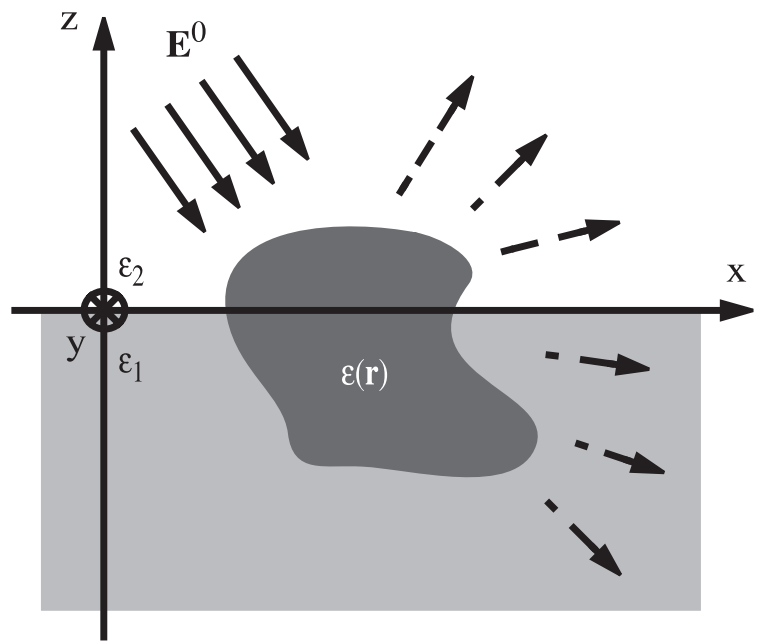

Fig. 1. Typical scattering problem considered in this work: the scatterer $\varepsilon(\mathbf{r})$ is deposited on, or embedded inside a surface of permittivity $\varepsilon_{1}$ and illuminated with an incident field $\mathbf{E}^{0}(\mathbf{r})$.

An analytic expression for the Green's tensor associated with a stratified medium does not exist and this dyadic must be computed numerically by means of Sommerfeld integrals [6-8]. However, when the scattering system is very small compared to the illumination wavelength, it should be possible to use a quasi-static formulation of the problem to simplify the computation.

The objective of this paper is to derive such a quasi-static formulation, and to assess its validity domain for practical scattering calculations. The formalism is developed in Section 2, where we first recall the computation of the Green's tensor for a stratified medium using Sommerfeld integrals and then derive the corresponding quasi-static expressions. We then compare in Section 3 this quasistatic approximation to the exact solution. In Section 3 we also analyze a global scattering problem using the quasi-static model. We make some concluding remarks in Section 4.

\section{Theory}

\subsection{Green's tensor for a multilayered system}

The type of structure that we consider in this article, namely a scatterer embedded in a two-layer system (Fig. 1), is a particular case of a multilayered medium. Therefore, we first discuss the general form of the Green's tensor for a multilayer structure; it is then an easy task to find the corresponding expressions for our two-layer structure.

An efficient way to evaluate the electromagnetic fields in a stratified medium made up of $N$ layers is to work in the spectral domain using the Hankel transform pair [7]:

$\tilde{g}\left(k_{\rho}\right)=\int_{0}^{\infty} J_{0}\left(k_{\rho} \rho\right) g(\rho) \rho \mathrm{d} \rho$,
$g(\rho)=\int_{0}^{\infty} J_{0}\left(k_{\rho} \rho\right) \tilde{g}\left(k_{\rho}\right) k_{\rho} \mathrm{d} k_{\rho}$,

where we use a "tilde" for the spectral domain. In this equation, $J_{0}$ is the zeroth-order Bessel function, $\rho=\sqrt{x^{2}+y^{2}}$, the transverse spatial coordinate and $k_{\rho}=\left(k_{x}^{2}+k_{y}^{2}\right)^{1 / 2}$, the corresponding spectral coordinate.

In the spectral domain, it is always possible to obtain analytical expressions for the normal components of the electric and magnetic fields $\tilde{G}_{z \alpha}^{\mathrm{E}}$, $\tilde{G}_{z \alpha}^{\mathrm{H}}(\alpha=x, y, z)$ and then derive all the other electromagnetic field components from them. Indeed, for each sourceless layer $i$ of the stratified medium, the normal component of the electromagnetic field $\psi_{i}$ is a solution of Helmholtz equation

$\left(\nabla^{2}+k_{i}^{2}\right) \psi_{i}=0$,

its transform $\tilde{\psi}_{i}$ being therefore a solution of the ordinary differential equation

$\left(\mathrm{d}^{2} / \mathrm{d} z^{2}-u_{i}^{2}\right) \tilde{\psi}_{i}=0$,

where $u_{i}=\left(k_{\rho}^{2}-k_{i}^{2}\right)^{1 / 2}$ is the normal propagation constant. A general solution of Eq. (4) can be written as

$\tilde{\psi}_{i}=a_{i} \cosh \left(u_{i} z\right)+b_{i} \sinh \left(u_{i} z\right)$,

where $a_{i}$ and $b_{i}$ are unknown complex coefficients. For the layer $e$ including the source, a particular solution of the form $c_{e} \exp \left(-u_{e}\left|z-z^{\prime}\right|\right)$ must be added to the general solution (5).

The coefficient $c_{e}$ depends on the source position and orientation, and on the field component to be evaluated. Expressions for $c_{e}$ can be found in Ref. [9]. Note that they should be divided by $-i \omega \mu$ 
in order to agree with the notation used in the present paper. The $2 N$ unknowns $a_{i}$, and $b_{i}$ are determined by enforcing the boundary conditions at each interface and by taking into account the Sommerfeld radiation condition, which states that the field originating at the source point must vanish for $z \rightarrow \pm \infty$. Further, the values of $u_{i}$ must be chosen in the fourth quadrant of the complex plane [10].

Let us apply this formalism to our two-layer system (Fig. 1). For brevity, we will only discuss in detail the case $z \geqslant 0$, and $z^{\prime}<0$, i.e., when the source is in the lower medium $\varepsilon_{1}$ and the observer in the upper medium $\varepsilon_{2}$. The results for other source-observer configurations are given in Appendix A. The spectral components of the vertical electric field reads

$\tilde{G}_{z \alpha}^{\mathrm{E}}=2 C_{\alpha}^{\mathrm{E}} \frac{u_{1} \varepsilon_{1}}{u_{2} \varepsilon_{1}+u_{1} \varepsilon_{2}} \mathrm{e}^{-u_{2} z} \mathrm{e}^{u_{1} z^{\prime}}, \quad \alpha=x, y, z$

with $C_{x}^{\mathrm{E}}=-\mathrm{i} k_{x} /\left(4 \pi k_{1}^{2}\right), \quad C_{y}^{\mathrm{E}}=-\mathrm{i} k_{y} /\left(4 \pi k_{1}^{2}\right) \quad$ and $C_{z}^{\mathrm{E}}=k_{\rho}^{2} /\left(4 \pi k_{1}^{2} u_{1}\right)$. The normal components of the magnetic field have a similar form as Eq. (6), with $\varepsilon$ replaced by $\mu$ and $C_{\alpha}^{\mathrm{E}}$ substituted by $C_{\alpha}^{\mathrm{H}}$, with $C_{x}^{\mathrm{H}}=k_{y} /\left(4 \pi \omega \mu_{0} u_{1}\right), \quad C_{y}^{\mathrm{H}}=-k_{x} /\left(4 \pi \omega \mu_{0} u_{1}\right) \quad$ and $C_{z}^{\mathrm{H}}=0$.

The transverse components of the dyadic Green's tensor can then be derived from the two vertical components $\tilde{G}_{z \alpha}^{\mathrm{E}}$ and $\tilde{G}_{z \alpha}^{\mathrm{H}}$ and from their normal derivatives $\partial \tilde{G}_{z \alpha}^{\mathrm{E}} / \partial z$ and $\partial \tilde{G}_{z \alpha}^{\mathrm{H}} / \partial z$. This leads to

$$
\begin{array}{ll}
\tilde{G}_{x \alpha}=\frac{1}{k_{\rho}^{2}}\left(\mathrm{i} k_{x} \frac{\partial \tilde{G}_{z \alpha}^{\mathrm{E}}}{\partial z}-k_{y} \omega \mu_{0} \tilde{G}_{z \alpha}^{\mathrm{H}}\right), & \alpha=x, y, z, \\
\tilde{G}_{y \alpha}=\frac{1}{k_{\rho}^{2}}\left(\mathrm{i} k_{y} \frac{\partial \tilde{G}_{z \alpha}^{\mathrm{E}}}{\partial z}+k_{x} \omega \mu_{0} \tilde{G}_{z \alpha}^{\mathrm{H}}\right), & \alpha=x, y, z .
\end{array}
$$

Note that Eqs. (7) and (8) are valid for any sourceobserver configuration. Similarly, we have an analytical expression for each spectral component of the electric field Green's tensor.

\subsection{Sommerfeld integrals}

To obtain the Green's tensor components in the space domain, as required by Eq. (1), we apply the inverse transform equation (2b). Unfortunately, no analytical expression corresponding to Eq. (6) can be found in the space domain and the fields must be computed numerically. The difficulties associated with such a numerical integration have been detailed by several authors [11,9]. The traditional integration path is the real axis of the complex spectral plane $[12,13]$. The imaginary axis can also be used to perform the numerical integration if one uses Hankel functions instead of Bessel functions [14]. In both the cases, the pole contributions are taken into account using the theory of residue. Another possibility is to select a path that avoids all the poles as proposed in Ref. [15]. Such a technique is particularly efficient when the structure is made up of many dielectric layers, rendering the search for the poles difficult.

The electric field Green's tensor associated with an $x$-oriented electric dipole is then given by

$$
\begin{aligned}
G_{x x}^{\mathrm{E}}= & -\frac{\cos (2 \varphi)}{\rho} \int_{0}^{\infty} J_{1}\left(k_{\rho} \rho\right)\left[\frac{\mathrm{i}}{k_{x}} \frac{\partial \tilde{G}_{z x}^{\mathrm{E}}}{\partial z}+\frac{\omega \mu_{0}}{k_{y}} \tilde{G}_{z x}^{\mathrm{H}}\right] \mathrm{d} k_{\rho} \\
& +\int_{0}^{\infty} J_{0}\left(k_{\rho} \rho\right) k_{\rho}\left[\cos ^{2}(\varphi) \frac{\mathrm{i}}{k_{x}} \frac{\partial \tilde{G}_{z x}^{\mathrm{E}}}{\partial z}\right. \\
& \left.-\sin ^{2}(\varphi) \frac{\omega \mu_{0}}{k_{y}} \tilde{G}_{z x}^{\mathrm{H}}\right] \mathrm{d} k_{\rho}, \\
G_{y x}^{\mathrm{E}}= & -\frac{\sin (2 \varphi)}{\rho} \int_{0}^{\infty} J_{1}\left(k_{\rho} \rho\right)\left[\frac{\mathrm{i}}{k_{x}} \frac{\partial \tilde{G}_{z x}^{\mathrm{E}}}{\partial z}+\frac{\omega \mu_{0}}{k_{y}} \tilde{G}_{z x}^{\mathrm{H}}\right] \mathrm{d} k_{\rho} \\
& +\frac{\sin (2 \varphi)}{2} \int_{0}^{\infty} J_{0}\left(k_{\rho} \rho\right) k_{\rho} \\
& \times\left[\frac{\mathrm{i}}{k_{x}} \frac{\partial \tilde{G}_{z x}^{\mathrm{E}}}{\partial z}+\frac{\omega \mu_{0}}{k_{y}} \tilde{G}_{z x}^{\mathrm{H}}\right] \mathrm{d} k_{\rho}, \\
G_{z x}^{\mathrm{E}}= & \cos \varphi \int_{0}^{\infty} J_{1}\left(k_{\rho} \rho\right) k_{\rho}^{2} \frac{\mathrm{i}}{k_{x}} \tilde{G}_{z x}^{\mathrm{E}} \mathrm{d} k_{\rho} .
\end{aligned}
$$

Expressions for $\tilde{G}_{\alpha y}^{\mathrm{E}}$ with $\alpha=x, y, z$, i.e, the components of the electric field Green's tensor associated with an $y$-oriented electric source can be readily obtained from Eq. (9a)-(9c) by replacing $\varphi$ with $\varphi-\pi / 2$.

Finally, for a vertical electric source, the components of Green's tensor are 


$$
\begin{aligned}
G_{x z}^{\mathrm{E}} & =-\cos \varphi \int_{0}^{\infty} J_{1}\left(k_{\rho} \rho\right) \frac{\partial \tilde{G}_{z z}^{\mathrm{E}}}{\partial z} \mathrm{~d} k_{\rho}, \\
G_{y z}^{\mathrm{E}} & =-\sin \varphi \int_{0}^{\infty} J_{1}\left(k_{\rho} \rho\right) \frac{\partial \tilde{G}_{z z}^{\mathrm{E}}}{\partial z} \mathrm{~d} k_{\rho}, \\
G_{z z}^{\mathrm{E}} & =\int_{0}^{\infty} J_{0}\left(k_{\rho} \rho\right) k_{\rho} \tilde{G}_{z z}^{\mathrm{E}} \mathrm{d} k_{\rho} .
\end{aligned}
$$

Sommerfeld integrals (9a)-(9c) and (10a)-(10c) have been implemented numerically and will be used as reference values in Section 3, since they proceed from a rigorous model.

\subsection{Quasi-static approximation}

The quasi-static approximation can be very useful for studying phenomena involving small scatterers. Indeed, the fields at short distances from a source are always quasi-static and can be evaluated by neglecting retardation, i.e., by formally setting $c=\infty$ in Maxwell's equations.

The quasi-static approximation arises from a systematic expansion of Maxwell's equations in power of $c^{-1}$ as explained in Ref. [16]. In our case, we can find the quasi-static Green's tensor in the vicinity of a dielectric surface by doing the quasistatic limit in the spectral domain: $k_{\rho} \rightarrow \infty$. Sommerfeld integrals (9a)-(9c) and (10a)-(10c) then take a simple form and can be solved analytically.

Let us illustrate this procedure by considering again the case $z \geqslant 0$ and $z^{\prime}<0$. Introducing Eq. (6) into Eq. (10c) and setting $k_{\rho} \rightarrow \infty$, we obtain

${ }^{*} G_{z z}^{\mathrm{E}}=\frac{2 \varepsilon_{2}}{4 \pi k_{2}^{2}\left(\varepsilon_{1}+\varepsilon_{2}\right)} \int_{0}^{\infty} J_{0}\left(k_{\rho} \rho\right) k_{\rho}^{2} \mathrm{e}^{-k_{\rho}\left(z-z^{\prime}\right)} \mathrm{d} k_{\rho}$,

where we use an asterisk for the quasi-static approximation of $G_{z z}^{\mathrm{E}}$. The integral in Eq. (11) can be solved using a generalization of the Lipschitz integral [17]. This leads to

$$
\begin{aligned}
{ }^{*} G_{z z}^{\mathrm{E}} & =\frac{2 \varepsilon_{2}}{4 \pi k_{2}^{2}\left(\varepsilon_{1}+\varepsilon_{2}\right)}\left[\frac{-1}{r^{3}}+\frac{3\left(z-z^{\prime}\right)^{2}}{r^{5}}\right] \\
& =\frac{2 \varepsilon_{2}}{\varepsilon_{1}+\varepsilon_{2}}{ }^{*} G_{z z}^{2},
\end{aligned}
$$

where $r=\left(\rho^{2}+\left(z-z^{\prime}\right)^{2}\right)^{1 / 2}$ and ${ }^{*} G_{z z}^{2}$ is simply the $z$-component of the electric field produced by a $z$ - oriented dipole located in an infinite homogeneous medium of permittivity $\varepsilon_{2}$. The quasi-static expression for each component of the electric field Green's tensor can be deduced from Eqs. (9a)-(9c) and (10a)-(10c). The surface Green's tensor has the form

${ }^{*} \mathbf{G}\left(\mathbf{r}, \mathbf{r}^{\prime}\right)=\frac{2 \varepsilon_{2}}{\varepsilon_{1}+\varepsilon_{2}} * \mathbf{G}^{2}\left(\mathbf{r}, \mathbf{r}^{\prime}\right)$,

where

${ }^{*} \mathbf{G}^{2}\left(\mathbf{r}, \mathbf{r}^{\prime}\right)=\left(\frac{-1}{k_{2}^{2} r^{2}} \mathbf{1}+\frac{3}{k_{2}^{2} r^{4}} \mathbf{R} \mathbf{R}\right) \frac{1}{4 \pi r}$.

For a source located under the surface, the quasistatic field above the surface is therefore given by the field associated with an infinite homogeneous medium weighted with a coefficient including the permittivity parameters of each layer.

For $z \geqslant 0$ and $z^{\prime} \geqslant 0$ (Fig. 2), the surface Green's tensor for the quasi-static case ${ }^{*} \mathbf{G}\left(\mathbf{r}, \mathbf{r}^{\prime}\right)$ is given by

${ }^{*} \mathbf{G}\left(\mathbf{r}, \mathbf{r}^{\prime}\right)={ }^{*} \mathbf{G}^{2}\left(\mathbf{r}, \mathbf{r}^{\prime}\right) \mp \frac{\varepsilon_{1}-\varepsilon_{2}}{\varepsilon_{1}+\varepsilon_{2}}{ }^{*} \mathbf{G}^{2}\left(\mathbf{r}, \mathbf{r}^{\prime \prime}\right)$,

where ${ }^{*} \mathbf{G}^{2}\left(\mathbf{r}, \mathbf{r}^{\prime}\right)$ is the quasi-static Green's tensor for an infinite homogeneous medium of permittivity $\varepsilon_{2}$. Note that ${ }^{*} \mathbf{G}^{2}\left(\mathbf{r}, \mathbf{r}^{\prime \prime}\right)$ takes into account the effect of an image source placed at $\mathbf{r}^{\prime \prime}$ (Fig. 2). In Eq. (15), the minus sign and the plus sign refer to

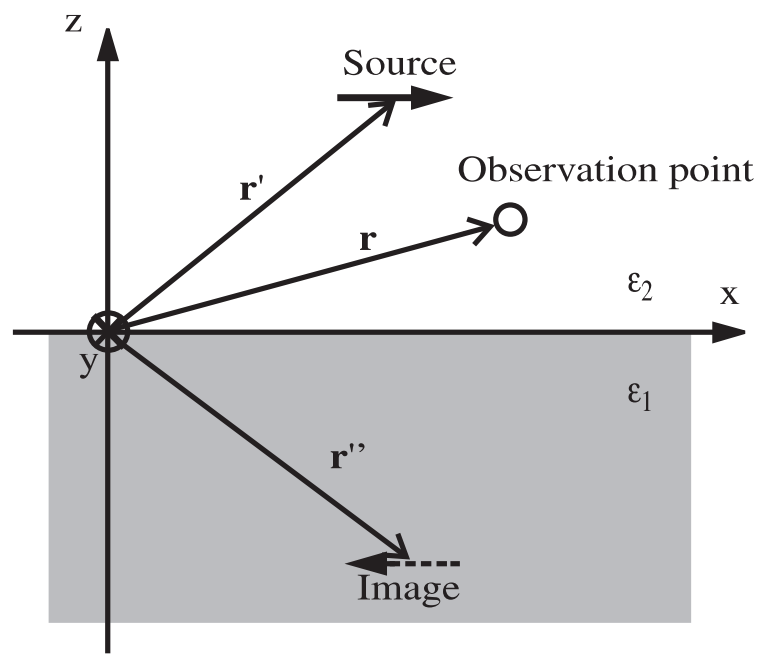

Fig. 2. Image location used for the quasi-static approximation. 
horizontal ( $x$-, $y$-directed) and vertical ( $z$-directed) sources, respectively.

For $z<0$ and $z^{\prime}<0$, the surface Green's tensor is given by

${ }^{*} \mathbf{G}\left(\mathbf{r}, \mathbf{r}^{\prime}\right)={ }^{*} \mathbf{G}^{1}\left(\mathbf{r}, \mathbf{r}^{\prime}\right) \pm \frac{\varepsilon_{1}-\varepsilon_{2}}{\varepsilon_{1}+\varepsilon_{2}} * \mathbf{G}^{1}\left(\mathbf{r}, \mathbf{r}^{\prime \prime}\right)$,

where ${ }^{*} \mathbf{G}^{1}\left(\mathbf{r}, \mathbf{r}^{\prime}\right)$ is the Green's tensor for an infinite homogeneous medium of permittivity $\varepsilon_{1}$. In this case, the plus sign and the minus sign are associated with horizontal and vertical sources, respectively.

Finally for $z<0$ and $z^{\prime} \geqslant 0$, the surface Green's tensor has the form

${ }^{*} \mathbf{G}\left(\mathbf{r}, \mathbf{r}^{\prime}\right)=\frac{2 \varepsilon_{1}}{\varepsilon_{1}+\varepsilon_{2}} * \mathbf{G}^{1}\left(\mathbf{r}, \mathbf{r}^{\prime}\right)$.

So, we have formally deduced the quasi-static approximation for the Green's tensor from a dynamic rigorous model expressed through Sommerfeld integrals. It is quite striking that our results are similar to that obtained by the image method.

\section{Discussion}

In this section, we study and discuss the validity domain and limitations of the non-retarded Green's tensor. First, we compare the non-retarded and retarded Green's tensors associated with a two-layer medium, for various surface permittivities and frequencies. Second, we use a series of practical scattering calculations to discuss the influence of retardation.

\subsection{Retarded and non-retarded Green's tensors}

For each definition, the quasi-static approximation is valid at small distances from the source, compared to the wavelength of emitted radiation. However, how small this distance should be to warrant the use of the quasi-static approximation is an open question. In order to quantify the validity region of this approximation, we compare the exact solutions for the electric field Green's tensor (evaluated from Sommerfeld integrals (9a)-

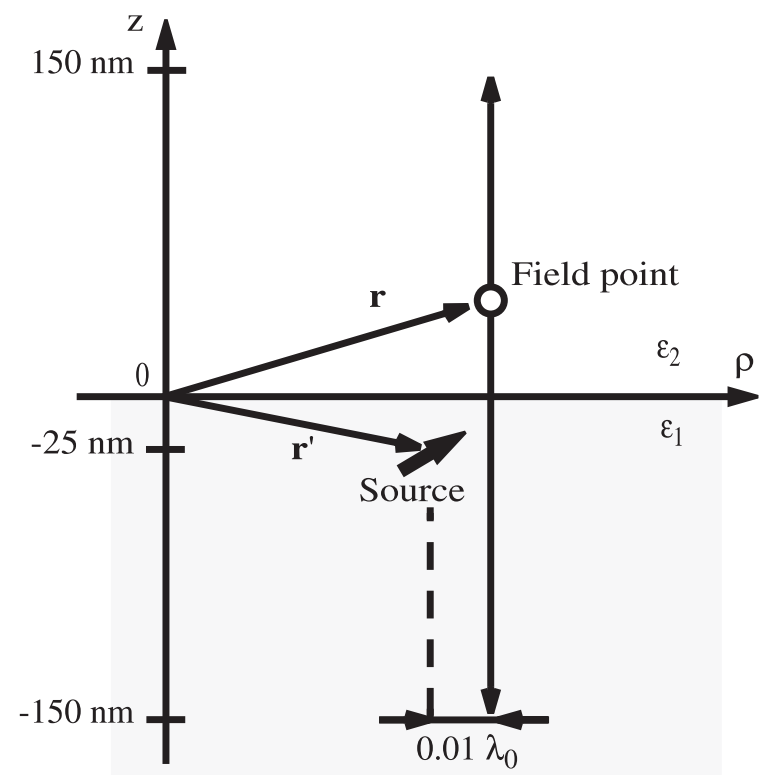

Fig. 3. Two-layer medium with a source located at $25 \mathrm{~nm}$ below the interface. The field displayed in Figs. 4 and 5 is calculated along the vertical line indicated in the figure.

(9c) and (10a)-(10c)) with the corresponding quasi-static approximations.

We consider a two-layer medium with an electric dipole inside the lower layer $\varepsilon_{1}=4$, located at $25 \mathrm{~nm}$ below the interface (Fig. 3). The permittivity $\varepsilon_{2}$ of the upper layer is 1 . We compute the Green's tensor components along the $z$-axis, from $-150 \mathrm{~nm}$ below the interface to $150 \mathrm{~nm}$ above, at a short transverse distance $\rho=0.01 \lambda_{0}$. In Fig. 4(a) and (b), we show the $x x$ - and $z x$-components of the Green's tensor for the two different wavelengths $\lambda_{0}=1000$ and $500 \mathrm{~nm}$. For each wavelength, a vertical dashed line is placed on either side of the source at an optical distance of $0.1 \lambda$ from the source (note that the effective wavelength is different in both media; therefore, the physical distances are different).

Up to an optical distance of $0.1 \lambda$ from the source, we observe a very good agreement between the exact and quasi-static curves. Moreover, the deviation of the quasi-static result from the exact solution has the same order of magnitude for two optically equidistant points. This can be observed in Fig. 4(a), considering, for instance, the two field 

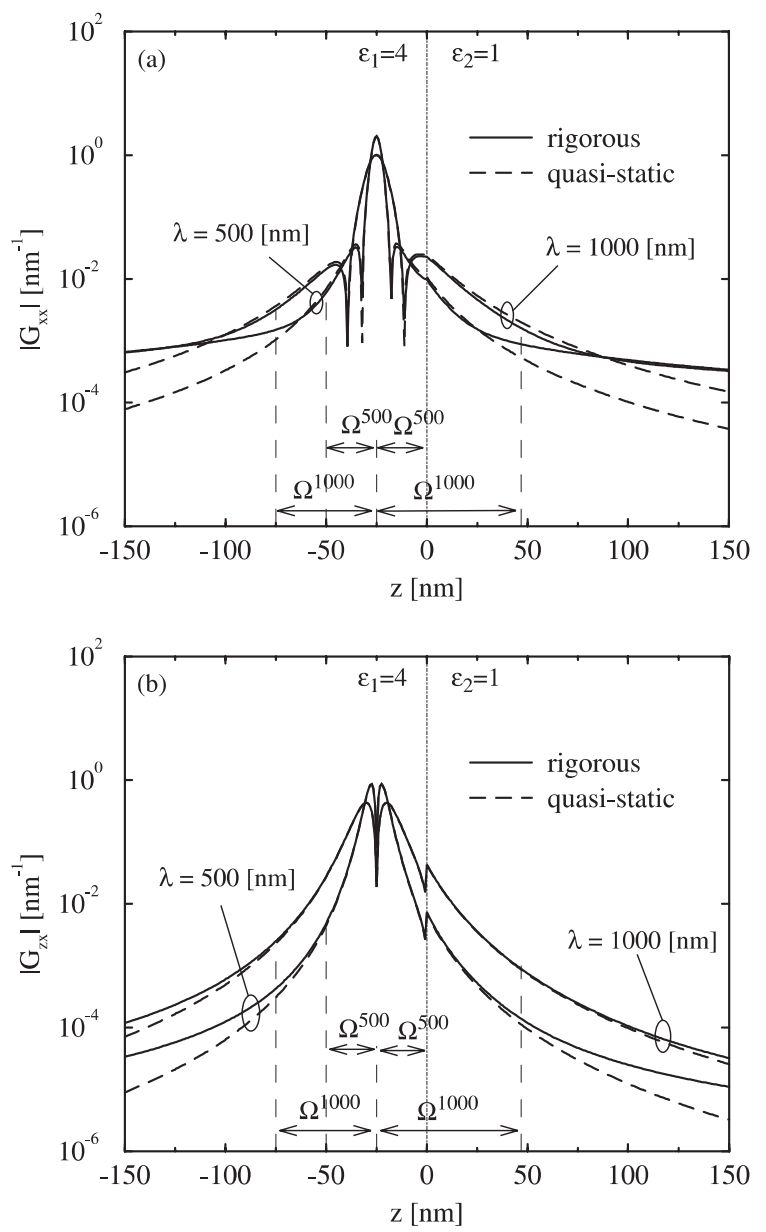

Fig. 4. Comparison between the rigorous and the quasi-static $x x$ - and $z x$-components of the Green's tensor for the geometry depicted in Fig. 3. The lower medium has $\varepsilon_{1}=4$ and the upper medium $\varepsilon_{2}=1$. Two different wavelengths $\lambda_{0}$ are considered.

points at $z \simeq 62 \mathrm{~nm}$ and $z \simeq 25 \mathrm{~nm}$ (for $\lambda_{0}=500$ $\mathrm{nm}$ ), which correspond to the region where the exact and approximated curves strongly diverge. A similar behavior is observed for the optically equidistant points $z=-100$ and $z \simeq 97 \mathrm{~nm}$ for $\lambda_{0}=1000 \mathrm{~nm}$.

This behavior is observed for each of the nine components of the electric field Green's tensor. Finally, it is quite striking that the discontinuity of the $z x$-component does not impinge on the accuracy of the quasi-static approximation.

In Fig. 5(a) and (b), we show the $x x$ - and $z x$ components of the Green's tensor for two different
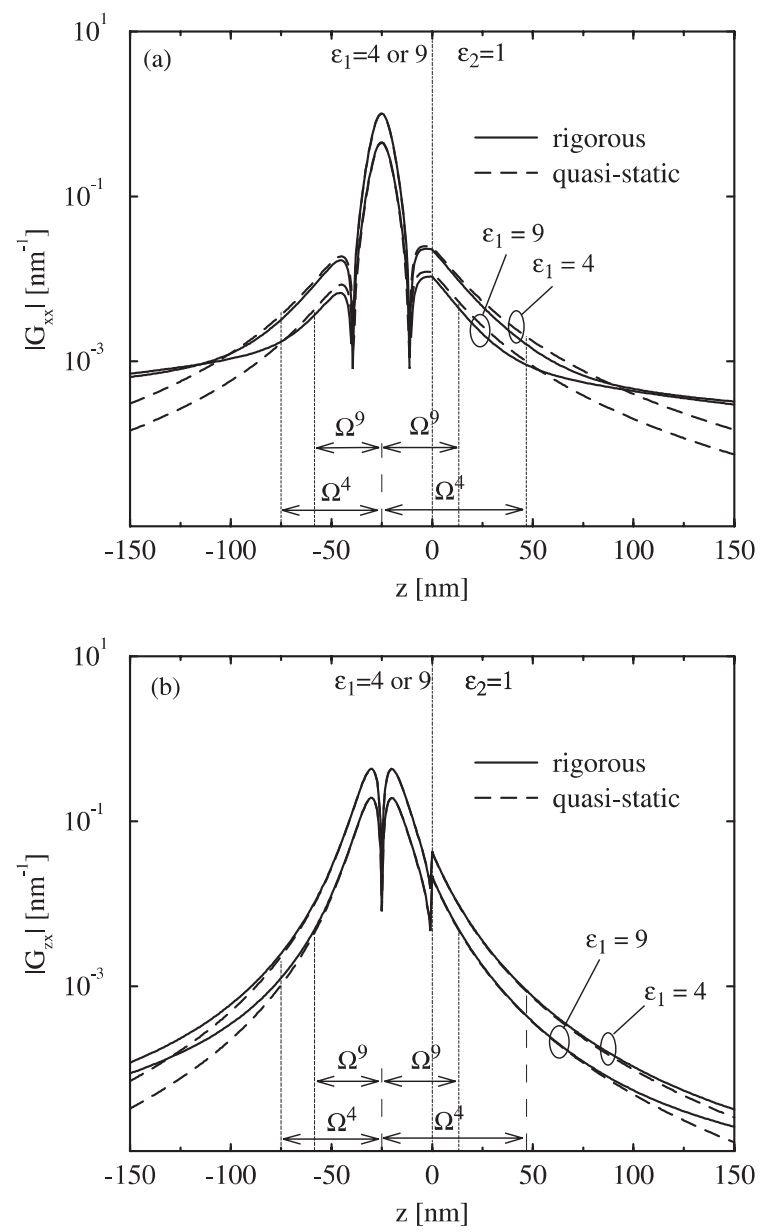

Fig. 5. Comparison between the rigorous and the quasi-static $x x$ - and $z x$-components of the Green's tensor for the geometry depicted in Fig. 3. The wavelength is $\lambda_{0}=1000 \mathrm{~nm}$ and two different lower media are considered: $\varepsilon_{1}=4$ and $\varepsilon_{1}=9$, while the upper medium is kept constant: $\varepsilon_{2}=1$.

values of the dielectric surface, with $\varepsilon_{1}=4$ and 9 . The illumination wavelength is $\lambda_{0}=1000 \mathrm{~nm}$. The study of the curves just confirms the remarks made above.

These results clearly demonstrate the validity of the electrostatic approximation, even in the presence of surfaces, for source-observation distances up to a 10 th of the effective wavelength.

\subsection{Analysis of a scattering problem}

In the previous section, we have seen that it is where the Green's tensor values are small that the 
quasi-static and rigorous models strongly diverge. For instance, we can observe in Fig. 4(a) that there is about three orders of magnitude between the maximum value of the function and the value where the divergence starts. The extent to which the quasi-static approximation will jeopardize a practical scattering calculation is not obvious, since the entire scattering might be dominated by short distance interaction, where the approximation is very good. To address this point, we investigate the case of a flat dielectric surface of permittivity $\varepsilon_{1}$ with a protrusion of permittivity $\varepsilon$ as shown in Fig. 6. We calculate the total electric field intensity in a plane parallel to the surface, located just above the protrusion, for various configurations. The main aim of this subsection is to compare the results obtained from the quasistatic and rigorous models (the details of the rigorous calculation can be found in Ref. [18]). We will always consider a protrusion of permittivity $\varepsilon=2.25$, an illumination wavelength in vacuum $\lambda_{0}=1000 \mathrm{~nm}$ and use a constant discretization mesh $\Delta x=\Delta y=\Delta z=5 \mathrm{~nm}$.

We first consider a protrusion of small dimensions $\mathrm{d} x=\mathrm{d} y=\mathrm{d} z=20 \mathrm{~nm}$ (Fig. 6), illuminated under evanescent field $\left(\theta=240^{\circ}\right)$. The permittivity values of the lower and upper medium are $\varepsilon_{1}=2.25$ and $\varepsilon_{2}=1$, respectively.

In Fig. 7, we show the field intensity distribution $5 \mathrm{~nm}$ above the protrusion computed with the quasi-static approximation. The incident field is $\mathrm{p}$ -

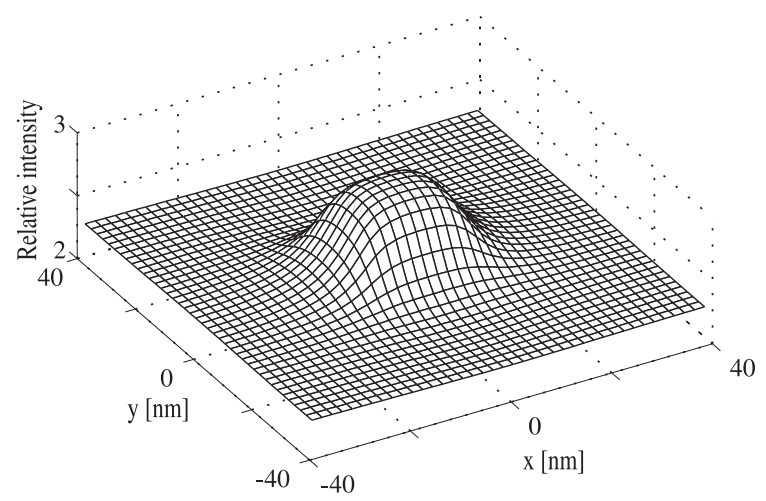

Fig. 7. Field intensity distribution $5 \mathrm{~nm}$ above a $\mathrm{d} x=\mathrm{d} y=$ $\mathrm{d} z=20 \mathrm{~nm}$ protrusion computed with the quasi-static approximation. The protrusion is deposited on a surface with permittivity $\varepsilon_{1}=2.25$. The illumination angle is $\theta=240^{\circ}$, and the incident field is p-polarized.

polarized. We observe the strong field confinement that reproduces the object shape, as discussed in detail in Ref. [19]. In Fig. 8(a), we show the relative error between the non-retarded (quasi-static) and retarded (rigorous) solutions. We can see a very good agreement between the reference and approximated values, the error being smaller than $10^{-5}$. Away from the scatterer, the error decreases, since the main contribution to the total field is the incident field transmitted through the interface, the scattered field becoming negligible. In Fig. 8(b) and (c), we show the relative error evaluated in the same plane for larger dimensions of the protrusion.

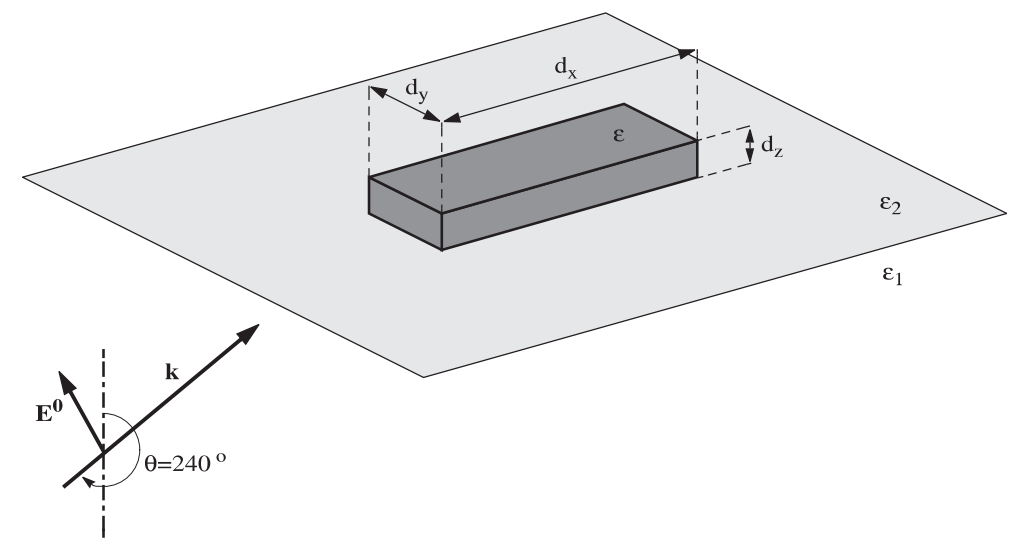

Fig. 6. Flat dielectric surface of permittivity $\varepsilon_{1}$ with a protrusion of index $\varepsilon$. The structure is illuminated with a p-or s-polarized incident plane wave. 

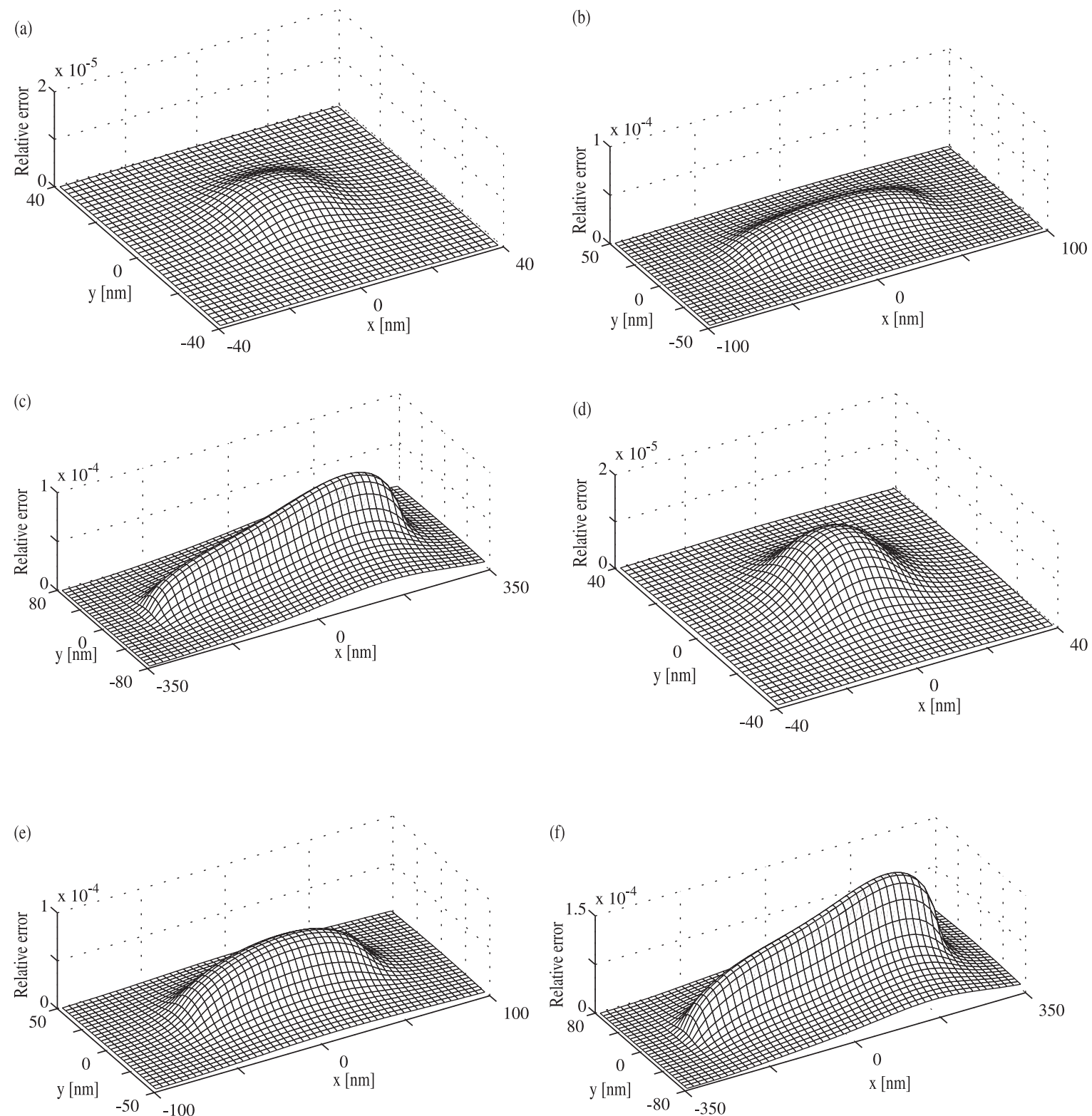

Fig. 8. Relative error between the quasi-static and rigorous solutions $5 \mathrm{~nm}$ above a protrusion $(\varepsilon=2.25)$ deposited on a surface. Three different protrusion dimensions are investigated: $(\mathrm{a}, \mathrm{d}): \mathrm{d} x=\mathrm{d} y=\mathrm{d} z=20 \mathrm{~nm},(\mathrm{~b}, \mathrm{e}): \mathrm{d} x=100 \mathrm{~nm}, \mathrm{~d} y=\mathrm{d} z=20 \mathrm{~nm}$, and $(\mathrm{c}, \mathrm{f})$ : $\mathrm{d} x=500 \mathrm{~nm}, \mathrm{~d} y=\mathrm{d} z=20 \mathrm{~nm}$. Two different substrate permittivities are considered: (a)-(c): $\varepsilon_{1}=2.25$, and (d)-(f): $\varepsilon_{1}=4$. The illumination angle is $\theta=240^{\circ}$, and the incident field p-polarized.

As expected, the error increases with the dimensions of the protrusion, but remains very small even for the highest permittivity.
From Fig. 8(d)-(f) we can note that a larger surface permittivity $\varepsilon_{1}$ increases the error (although the protrusion keeps the same permittivity 

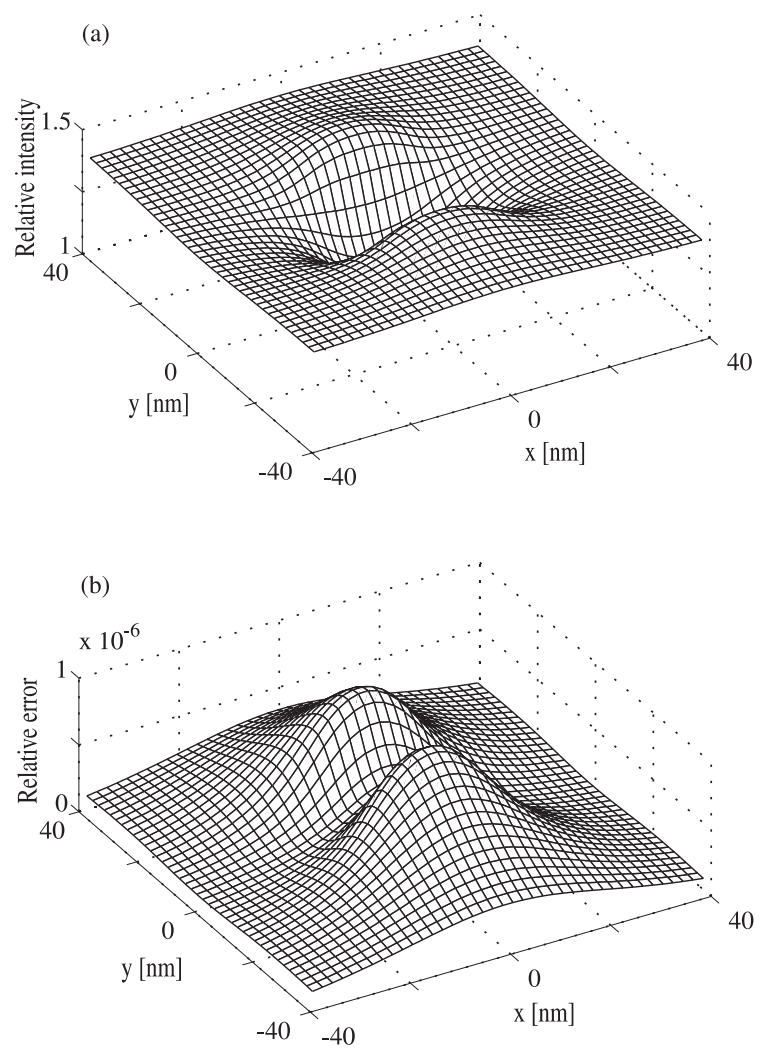

Fig. 9. (a) Field intensity distribution computed with the quasistatic approximation and (b) relative error between quasi-static and rigorous solutions computed $5 \mathrm{~nm}$ above a $\varepsilon=2.25$, $\mathrm{d} x=\mathrm{d} y=\mathrm{d} z=20 \mathrm{~nm}$ protrusion deposited on a surface with permittivity $\varepsilon_{1}=2.25$. The illumination angle is $\theta=240^{\circ}$, and the incident field s-polarized.

$\varepsilon=2.25)$. Then, we then consider an s-polarized incident field, and present in Fig. 9(a), the field intensity distribution and in Fig. 9(b), the relative error between quasi-static and rigorous solutions for a protrusion of dimensions $\mathrm{d} x=\mathrm{d} y=\mathrm{d} z=20$ nm. We can observe in particular that the field intensity makes the object appear in reversed contrast as found in Ref. [19]. The relative error is very small again, the maximum value being less than $10^{-6}$. We have also verified that, as for $\mathrm{p}$ polarization, the relative error increases with the dimensions of the protrusion and the permittivity of the dielectric surface.

We further consider the relative error associated with the same configuration under another illu- mination angle $\left(\theta=300^{\circ}\right)$ for two different permittivities of the dielectric surface $\varepsilon_{1}=2.25$ (Fig. 10(a)) and $\varepsilon_{1}=4$ (Fig. 10(b)). Relative error in both cases retains the same order of magnitude as in the previous case.

Finally, having verified that the near-field is accurately reproduced using the quasi-static approximation, we were interested in the field further away from the scatterer. In Fig. 11, we report the field intensity and the relative error above the center of the protrusion, as a function of the distance. Note the stationary wave created by the interference of the incident and reflected fields and the rapid decrease of the error with the distance is to be noted.
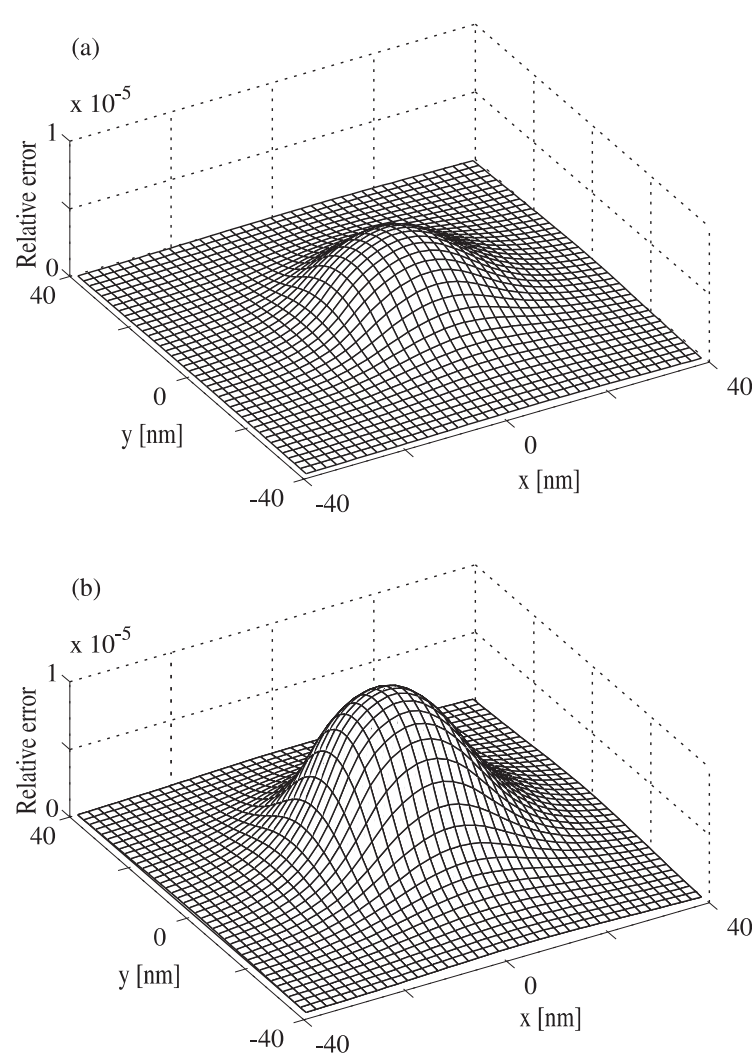

Fig. 10. Relative error between the quasi-static and rigorous solutions $5 \mathrm{~nm}$ above a $\mathrm{d} x=\mathrm{d} y=\mathrm{d} z=20 \mathrm{~nm}$ protrusion. The protrusion is deposited on a surface with permittivity (a) $\varepsilon_{1}=2.25$ and (b) $\varepsilon_{1}=4$. The illumination angle is $\theta=300^{\circ}$, and the incident field p-polarized. 


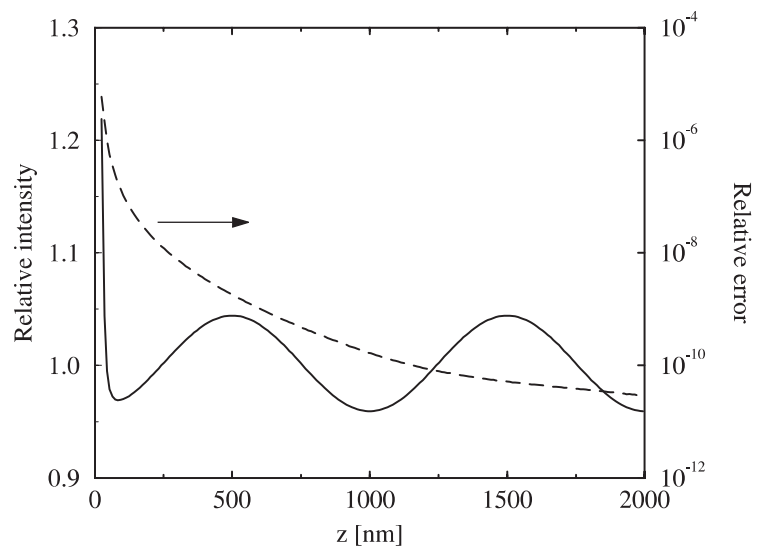

Fig. 11. Field intensity distribution computed with the quasistatic approximation and relative error between the quasi-static and rigorous solutions as a function of the distance $z$ above the scatterer. The protrusion has $\varepsilon=2.25, \mathrm{~d} x=\mathrm{d} y=\mathrm{d} z=20 \mathrm{~nm}$ and is deposited on a surface with permittivity $\varepsilon_{1}=2.25$. The illumination angle is $\theta=300^{\circ}$, and the incident field p-polarized.

\section{Conclusion}

In this paper, we have formally deduced the quasi-static approximation for the Green's tensor associated with a two-layer medium. The derivation has been performed from a rigorous model expressed in terms of Sommerfeld integrals.

The quasi-static model is very useful to compute the Green's tensor because it is much more rapid, since it is analytical and does not require the computation of complicated Sommerfeld integrals. The computation time of the rigorous Green's tensor strongly depends on the source-observer configuration, typical values are 400 CPUs for $1000\left(\mathbf{r}, \mathbf{r}^{\prime}\right)$ pairs on an IBM RS/6000 7012-397, $160 \mathrm{MHz}$ system [10]. Using the quasi-static model, the CPU time is only 1 CPUs for the same calculation.

The validity domain of the quasi-static approximation is solely determined by the effective wavelength of radiation used and our results show that within a 10th of this effective wavelength, the agreement between the quasi-static and the rigorous Green's tensors is perfect.

Moreover, with the study of a several scattering problem, we have demonstrated that the quasi- static approximation could be successfully used to investigate scatterers with dimensions up to one wavelength on low permittivity substrates.

\section{Acknowledgements}

It is a pleasure to acknowledge stimulating discussions with A. Dereux, C. Girard and M. Paulus. This work was supported by the Swiss National Science Foundation.

\section{Appendix A}

Here, we give the spectral components of the vertical electric field for a two-layer medium as shown in Fig. 1. Different cases must be distinguished, depending on the relative position $z^{\prime} / z$ of the source/observer points.

\section{A.1. Case $z^{\prime}<0$}

(a) For $z \geqslant 0$, see Eq. (6).

(b) For $z^{\prime} \leqslant z<0$,

$$
\begin{aligned}
G_{z(x, y, z)}^{\mathrm{E}}= & 2 C_{(x, y, z)}^{\mathrm{E}} \exp \left(u_{1} z^{\prime}\right) \\
& \times\left[\frac{u_{1} \varepsilon_{2} \cosh \left(u_{1} z\right)-u_{2} \varepsilon_{1} \sinh \left(u_{1} z\right)}{u_{1} \varepsilon_{2}+u_{2} \varepsilon_{1}}\right] .
\end{aligned}
$$

(c) For $z<z^{\prime}$,

$$
\begin{aligned}
G_{z(x, y)}^{\mathrm{E}}= & 2 C_{(x, y)}^{\mathrm{E}} \exp \left(u_{1} z\right) \\
& \times\left[\frac{u_{1} \varepsilon_{2} \sinh \left(u_{1} z^{\prime}\right)-u_{2} \varepsilon_{1} \cosh \left(u_{1} z^{\prime}\right)}{u_{1} \varepsilon_{2}+u_{2} \varepsilon_{1}}\right],
\end{aligned}
$$

$$
\begin{aligned}
G_{z z}^{\mathrm{E}}= & 2 C_{z}^{\mathrm{E}} \exp \left(u_{1} z\right) \\
& \times\left[\frac{u_{1} \varepsilon_{2} \cosh \left(u_{1} z^{\prime}\right)-u_{2} \varepsilon_{1} \sinh \left(u_{1} z^{\prime}\right)}{u_{1} \varepsilon_{2}+u_{2} \varepsilon_{1}}\right],
\end{aligned}
$$

where the coefficients $C_{(x, y, z)}^{\mathrm{E}}$ are defined below Eq. (6). 


\section{A.2. Case $z^{\prime} \geqslant 0$}

(a) For $z \geqslant z^{\prime}$,

$$
\begin{aligned}
G_{z(x, y)}^{\mathrm{E}}= & -2 C_{(x, y)}^{\mathrm{E}} \exp \left(-u_{2} z\right) \\
& \times\left[\frac{u_{2} \varepsilon_{1} \sinh \left(u_{2} z^{\prime}\right)+u_{1} \varepsilon_{2} \cosh \left(u_{2} z^{\prime}\right)}{u_{2} \varepsilon_{1}+u_{1} \varepsilon_{2}}\right],
\end{aligned}
$$

$G_{z z}^{\mathrm{E}}=2 C_{z}^{\mathrm{E}} \exp \left(-u_{2} z\right)$

$$
\times\left[\frac{u_{2} \varepsilon_{1} \cosh \left(u_{2} z^{\prime}\right)+u_{1} \varepsilon_{2} \sinh \left(u_{2} z^{\prime}\right)}{u_{2} \varepsilon_{1}+u_{1} \varepsilon_{2}}\right] .
$$

(b) For $z^{\prime}>z \geqslant 0$,

$$
\begin{aligned}
G_{z(x, y, z)}^{\mathrm{E}}= & 2 C_{(x, y, z)}^{\mathrm{E}} \exp \left(-u_{2} z^{\prime}\right) \\
& \times\left[\frac{u_{2} \varepsilon_{1} \cosh \left(u_{2} z\right)+u_{1} \varepsilon_{2} \sinh \left(u_{2} z\right)}{u_{2} \varepsilon_{1}+u_{1} \varepsilon_{2}}\right] .
\end{aligned}
$$

(c) For $z<0$,

$$
\begin{aligned}
\tilde{G}_{z(x, y, z)}^{\mathrm{E}}= & 2 C_{(x, y, z)}^{\mathrm{E}} \frac{u_{2} \varepsilon_{2}}{u_{2} \varepsilon_{1}+u_{1} \varepsilon_{2}} \exp \left(u_{1} z\right) \\
& \times \exp \left(-u_{2} z^{\prime}\right)
\end{aligned}
$$

where $C_{x}^{\mathrm{E}}=\mathrm{i} k_{x} /\left(4 \pi k_{2}^{2}\right), C_{y}^{\mathrm{E}}=\mathrm{i} k_{y} /\left(4 \pi k_{2}^{2}\right)$ and $C_{z}^{\mathrm{E}}=$ $k_{\rho}^{2} /\left(4 \pi k_{2}^{2} u_{2}\right)$.

\section{References}

[1] E.M. Purcell, C.R. Pennypacker, Astrophys. J. 186 (1973) 705.

[2] G.H. Goedecke, S.G. O’Brien, Appl. Opt. 27 (1988) 2431.

[3] W.T. Doyle, Phys. Rev. B 39 (1989) 9852.

[4] B.T. Draine, P.J. Flatau, J. Opt. Soc. Am. A 11 (1994) 1491.

[5] P.J. Flatau, Opt. Lett. 22 (1997) 1205.

[6] A. Sommerfeld, Partielle Differentialgleichungen der Physik, Geest and Portig, Leipzig, 1947.

[7] J.A. Stratton, Electromagnetic Theory, McGraw-Hill, New York, 1941.

[8] C.-T. Tai, Dyadic Green function in electromagnetic theory, IEEE Press, New York, 1994.

[9] J.R. Mosig, Numerical techniques for microwave and millimeter-wave passive structures, in: T. Itoh (Ed.), Integral Equation Technique, Wiley, New York, 1989, pp.133-213 (Chapter 3).

[10] M. Paulus, P. Gay-Balmaz, O.J.F. Martin, Phys. Rev. E in press (2000).

[11] L.B. Felsen, N. Marcuvitz, Radiation and Scattering of Waves, IEEE Press, New York, 1994.

[12] N.K. Uzunoglu, N.G. Alexopoulos, J.G. Fikioris, IEEE Trans. Antennas Propag., 1979.

[13] L. Barlatey, J.R. Mosig, T. Sphicopoulos, IEEE Trans. Antennas Propag. 38 (1990) 608.

[14] J.R. Mosig, A. Alvarez-Melcon, IEEE Antennas Propag. Soc. Int. Symp. IEEE, New York, vol. 1, 1996, pp. 416419.

[15] P. Gay-Balmaz, J.R. Mosig, Int. J. Microwave Millimeter Wave CAE 3 (1997) 330.

[16] G.W. Ford, W.H. Weber, Phys. Rep. 113 (1984) 195.

[17] G.N. Watson, A treatise on the theory of Bessel functions, Cambridge University Press, Cambridge, MA, 1944.

[18] M. Paulus, O.J.F. Martin, J. Opt. Soc. Am. A submitted for publication (2000).

[19] O.J.F. Martin, C. Girard, A. Dereux, J. Opt. Soc. Am. A 13 (1996) 1801. 